\title{
Seltene Erkrankungen und Hals-Nasen-Ohren-Heilkunde, Kopf und Halschirurgie
}

\author{
Rare Diseases and Otorhinolaryngology, Head and \\ Neck Surgery
}

\section{(ㄷ) (i) (우)}

Autor

Stefan K. Plontke

Institut

Universitätsklinik und Poliklinik für Hals-Nasen-Ohren-Heilkunde, Kopf- und Hals-Chirurgie Martin-Luther-Universität Halle-Wittenberg, Halle (Saale)

\section{Schlüsselwörter}

Seltene Erkrankungen, Hals-Nasen-Ohren-Heilkunde, Kopfund Hals-Chirurgie, Medizinethik, Orphan-Arzneimittel

\section{Key words}

rare diseases, otorhinolaryngology, medical ethics, orphan drugs

\section{Bibliografie}

Laryngo-Rhino-Otol 2021; 100: S1-S11

DOI 10.1055/a-1397-0842

ISSN 0935-8943

(c) 2021. The Author(s).

This is an open access article published by Thieme under the terms of the Creative Commons Attribution-NonDerivative-NonCommercial-License, permitting copying and reproduction so long as the original work is given appropriate credit. Contents may not be used for commecial purposes, or adapted, remixed, transformed or built upon. (|s://creativecommons.org/ licenses/by-nc-nd/4.0/)

Georg Thieme Verlag KG, Rüdigerstraße 14,

70469 Stuttgart, Germany

Korrespondenzadresse

Prof. Dr. med. Stefan K. Plontke

Univ. HNO-Klinik

Ernst-Grube-Straße 40

D-06120 Halle/S.

stefan.plontke@uk-halle.de

Tel.: + 49 (0) 345 / 557 1840, Fax: 49 (0) 345 / 5571859

\section{ZUSAMMENFASSUNG}

Seltene Erkrankungen stellen betroffene Patienten, ihre Angehörigen, Ärzte, Pflegekräfte und Therapeuten oft vor besonde- re Herausforderungen. Ihre Seltenheit erschwert aus medizinischen und ökonomischen Gründen häufig die Forschung und die medizinische Versorgung. Viele typische Krankheiten im HNO-Fachgebiet zählen definitionsgemäß allein aufgrund ihrer Prävalenz zu den seltenen Erkrankungen. Die Einleitung der richtigen Schritte zum Management dieser Patienten setzt Kenntnisse über die Diagnostik, über bestehende Ressourcen wie Zentren, Netzwerke und Register, die Besonderheiten in der Arzt-Patienten-Beziehung, die Nachsorge einschließlich der Kommunikation mit den betreuenden Hausärzten und die Rolle von Selbsthilfegruppen voraus. Von besonderem Interesse für die Universitätsmedizin und die wissenschaftliche Fachgesellschaft sind die Besonderheiten im Bereich der Forschung einschließlich der europäischen Vernetzung und Forschungsförderung, des Informationsmanagements, der Öffentlichkeitsarbeit, der Aus-, Fort- und Weiterbildung, Aspekte der Finanzierung sowie regulatorische Aspekte, wie Orphan Drugs und klinische Studien bei kleinen Populationen.

\section{ABSTRACT}

Rare diseases pose multiple challenges for patients, relatives, physicians, nursing staff, and therapists. Their rarity impedes research and treatments due to medical and economical reasons. Many diseases in the field otorhinolaryngology, head and neck surgery are rare diseases due to their low prevalence. The initiation of the right management processes requires knowledge about diagnostics, resources like centers, networks and registries, about specifics of the physician-patient relationship, follow-up care including communication with family doctors and the role of self-help groups. Of special interest for university hospitals and our scientific society are the specific aspects of research including European networks and research funding, information management, public relations, education, training, financing, and regulations like orphan drugs and clinical trials in small populations. 


\section{Inhaltsverzeichnis}

Abstract

1. Einführung

2. Definition

3. Orphanet

4. Orphan-Arzneimittel („Orphan Drugs“)

5. Klinische Studien und biometrische Aspekte

6. Ethische Aspekte

7. Juristische Aspekte und ambulante spezialfachärztliche Versorgung

8. Nationales Aktionsbündnis für Menschen mit seltenen Erkrankungen (NAMSE)

9. Allianz Chronischer seltener Erkrankungen - ACHSE e.V

10. Forschung und Forschungsförderung

11. Fazit

Danksagung

Interessenkonflikt

Literatur
Viele typische Erkrankungen in unserem Fachgebiet zählen definitionsgemäß allein aufgrund ihrer geringen Prävalenz zu den seltenen Erkrankungen. Aufgrund der Charakteristik unseres Fachgebietes, welches sich v. a. über die Körperregion (Kopf und Hals) definiert und dadurch als Querschnittsfachgebiet mannigfaltige Berührungs- und Überschneidungspunkte mit anderen Fachgebieten aufweist, kommen Hals-Nasen-Ohren-Ärzte zwangsläufig mit Manifestationen seltener Erkrankungen in Kontakt. Es ist auch nicht ungewöhnlich, dass sich seltene Erkrankungen im HNO-Gebiet erst manifestieren.

Dadurch entsteht die Notwendigkeit, dass sich HNO-Ärzte intensiv mit dem Thema der seltenen Erkrankungen in unserem Fachgebiet auseinandersetzen. Dies schließt Kenntnisse über die Diagnostik, über bestehende Ressourcen wie Zentren, Netzwerke und Register, die Besonderheiten in der Arzt-Patienten-Beziehung, die Nachsorge einschließlich der Kommunikation mit den betreuenden Hausärzten und die Rolle von Selbsthilfegruppen voraus. Von besonderem Interesse für die Universitätsmedizin und unsere wissenschaftliche Fachgesellschaft sind die Besonderheiten im Bereich der Forschung einschließlich der europäischen Vernetzung und Forschungsförderung, des Informationsmanagements, der Öffentlichkeitsarbeit, der Aus-, Fort- und Weiterbildung, Aspekte der Finanzierung sowie regulatorische Aspekte (Orphan-Drugs und klinische Studien).

\section{Definition}

Die konkrete Definition für seltene Erkrankungen (seltene Krankheiten, „Rare Diseases“, „Orphan Diseases“) unterscheidet sich von Land zu Land. In der Europäischen Union (EU) gilt eine Erkrankung als selten, wenn nicht mehr als 5 von 10000 Menschen (50/105 bzw. 1:2000 Menschen) von ihr betroffen sind. Die US-amerikanischen Behörden verwenden eine Prävalenz von höchstens 1:1500 Menschen (7,5 pro $10000 \mathrm{EW}$ ), Japan 1:2500 (4 pro $10000 \mathrm{EW}$ ), Australien 1:10000 und die Schweiz sogar nur von 1:100000 Menschen [2,3].

Auch in der EU betreffen die meisten der seltenen Erkrankungen nur eine Person oder weniger pro 100000 EW. Ungefähr 5000 bis 8000 einzelne unterschiedliche seltene Erkrankungen betreffen jedoch zusammen eine Gesamtzahl von 27 bis 36 Mio. Menschen in der EU, d. h. 6-8\% der EU-Population. In Deutschland leben damit etwa circa 4 bis 6 Mio. Menschen mit einer seltenen Erkrankung.

Der Begriff „Orphan“ wird auch in Zusammenhang mit Krankheiten und Therapien („Orphan Drugs“) verwendet, die an sich häufig sind (z. B. viele Tropenkrankheiten), aber unter anderem aufgrund ökonomischer Ursachen, einschließlich des Fehlens von wirtschaftlichen Anreizen zur Entwicklung von Therapien, vernachlässigt werden. Diese in ärmeren Ländern typischen Krankheiten sollen aber nicht Gegenstand dieses Artikels sein.

\section{Orphanet}

Orphanet ist eine Datenbank, die Ressourcen zu seltenen Krankheiten und Medikamenten zur Behandlung seltener Krankheiten (sogenannter orphan drugs) bündelt, mit dem Ziel „das Wissen um seltene Krankheiten zu sammeln und zu erweitern, um so die Diagnose, Versorgung und Behandlung von Patienten mit seltenen Krankheiten zu verbessern. Orphanet stellt hochwertige Informationen über seltene Krankheiten zur Verfügung und gewährt gleich- 
berechtigten Zugriff auf diese Informationen für alle Interessengruppen. Orphanet pflegt darüber hinaus die Orphanet-Nomenklatur der seltenen Krankheiten (ORPHA code), die wesentlich zur Verbesserung der Sichtbarkeit von seltenen Krankheiten in Informationssystemen von Gesundheits- und Forschungseinrichtungen beiträgt“ [4].

Orphanet wurde im Jahr 1997 durch das französische Gesundheitsministerium und das „Institut national de la santé et de la recherche médicale“ (INSERM, Französisches nationales Institut für Gesundheit und medizinische Forschung) initiiert. Seit dem Jahr 2000 wird die Initiative von einem Konsortium mit inzwischen 40 Partnerländern aus Europa und weiteren internationalen Mitgliedern unter französischer Federführung und finanzieller Förderung durch die Europäische Union betrieben.

Orphanet, das Portal für seltene Krankheiten und orphan drugs bietet:

- ein Inventar, eine Klassifikation und eine Enzyklopädie der seltenen Krankheiten mit assoziierten Genen,

- Informationen über Arzneimittel für seltene Krankheiten (orphan drugs),

- ein Verzeichnis der Selbsthilfeorganisationen,

- ein Verzeichnis von Fachleuten und Einrichtungen sowie Expertenzentren,
- ein Verzeichnis medizinischer Labors, die diagnostische Leistungen anbieten,

- ein Verzeichnis der laufenden Forschungsprojekte klinischer Studien, Register und Biobanken sowie

- eine Sammlung der Themenartikel (Orphanet-Berichtsreihe) [4, 5], (> Abb. 1).

In der Orphanet-Datenbank kann nach Krankheiten, klinischen Zeichen und Symptomen, Klassifikationen, Genen und Behinderungen (Aktivitätseinschränkungen/Beeinträchtigungen des täglichen Lebens bzw. funktionelle Konsequenzen), die mit seltenen Krankheiten assoziiert sein können, gesucht werden. Der funktionelle Orphanet-Thesaurus basiert auf der ICF-Klassifikation der Weltgesundheitsorganisation (International Classification of Functioning, Disability and Health - Children and Youth, ICF-CY, WHO 2007).

Die Orphanet-Nomenklatur beinhaltet dabei eine „heterogene Typologie von Entitäten in der folgenden abnehmenden Rangfolge: Gruppen von Krankheiten, Krankheiten, Subtypen. Eine Krankheit der Orphanet-Datenbank entspricht einer der folgenden Definitionen: einer Erkrankung, einem Fehlbildungs-Syndrom, einem klinischen Syndrom, einer morphologischen oder biologischen Anomalie oder einer besonderen klinischen Situation (im Verlauf einer Erkrankung). Die Krankheiten sind in Gruppen organisiert und dann

\title{
Das Portal für seltene Krankheiten und Orphan Drugs
}

\author{
„Keine Krankheit kann zu selten sein, \\ um ihr Aufmerksamkeit zu schenken "
}

\section{Nutzen Sie Orphanet}
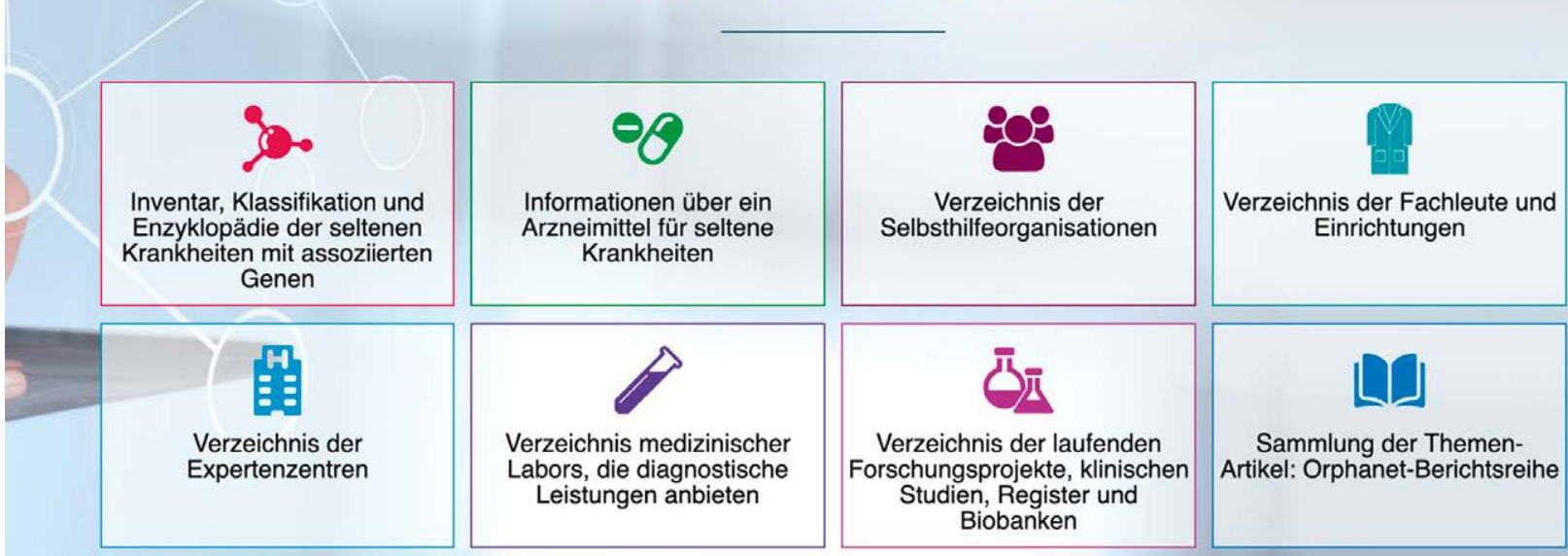

Q Bitte Krankheit eingeben

Suche

- Abb. 1 Eröffnungsseite der Datenbank Orphanet mit verfügbaren Ressourcen zu seltenen Krankheiten und Orphan Drugs (www.orpha.net) 
in klinische, ätiologische und histopathologische Subtypen unterteilt" [4].

Bei der Suche können unter anderem der Name der gesuchten Krankheit, der ORPHAcode, das Gen-Symbol/der Gen-Name, die OMIM- bzw. MIM-Nummer („Online Mendelian Inheritance in Man“, eine Datenbank, in der Gene des Menschen und deren Mutationen erfasst sind) oder der ICD-10-Code (International Classification of Diseases) als Suchkriterien eingegeben werden.

Orphanet stellt auch eine Enzyklopädie der seltenen Krankheiten für Fachleute zur Verfügung. Die Enzyklopädie für Fachleute beinhaltet folgende, von Experten erstellte und einer fachlichen Begutachtung unterliegenden Informationen: „Review-Artikel, klinische Leitlinien, Diagnosekriterien, Leitlinien für Gentestanalysen, das, European Journal of Human Genetics“, „Practical Genetics“, Klinische Genetik, Notfall-Leitlinien, Anästhesie-Leitlinien, Merkblätter über Behinderungen und zusammenfassende Kurzbeschreibungen von Krankheiten. Diese Texte können in den 7 Sprachen der Datenbank und darüber hinaus in weiteren Sprachversionen verfügbar sein" [4].

Für Behandler und Patienten stellt Orphanet Informationen über Selbsthilfegruppen (Patientenorganisationen, Dachverbände und Allianzen), die sich auf eine bestimmte Krankheit oder auf eine Gruppe von Krankheiten spezialisieren, auch geographisch unterteilt (nach Land, Region oder Stadt), zur Verfügung.

(Erst) seit 2019 besteht eine Kooperation zwischen dem „International Clinical Trials Registry Platform“ (ICTRP) - Register der Weltgesundheitsorganisation (https://www.who.int/ictrp/en/) und Orphanet. Mit dem Orphanet-Register wird die Identifikation klinischer Studien zu seltenen Krankheiten für ICTRP-Anwender deutlich verbessert (http://apps.who.int/trialsearch/).

Mit der „Orphanet Berichtsreihe“ erstellt und veröffentlicht Orphanet eine Reihe von als Download verfügbaren Berichten mit unterschiedlichen Schwerpunkten, die sich inhaltlich mit der Gesamtheit aller seltenen Krankheiten auseinandersetzen. Diese umfassen folgende "Themen":

- das Gesamtverzeichnis der seltenen Krankheiten,

- einen epidemiologischen Bericht mit verfügbaren Prävalenzdaten,

- das Verzeichnis der Orphan Drugs,

- einen Überblick der verfügbaren Register für seltene Krankheiten in Europa,

- den jährlichen Tätigkeitsbericht,

- einen Bericht mit einem Verzeichnis relevanter (Forschungs-) Infrastrukturen in Europa und

- die Ergebnisse der Orphanet-Umfrage zur Nutzerzufriedenheit und eine Liste aller Experten, die zu den Inhalten der Orphanet Datenbank beigetragen haben." (zitiert aus: [4]).

Die offizielle Zeitschrift von Orphanet ist das im Jahr 2006 gegründete „Orphanet Journal of Rare Diseases“, welches von BioMed Central (SpringerNature) „Open-Access“ veröffentlicht wird. Es enthält Publikationen zur Forschung bei seltenen Krankheiten im Rahmen von Übersichtsarbeiten und klinischen Studien sowie Artikel zum öffentlichen Gesundheitswesen („Public Health") und zu Arzneimitteln für seltene Krankheiten.

\section{Orphan-Arzneimittel („Orphan Drugs“)}

Als „Orphan-Arzneimittel“ („orphan“: engl. „Die Waise“) werden seit 1983 Medikamente bezeichnet, die für die Behandlung seltener Krankheiten eingesetzt werden. Bei diesen „Arzneimitteln für seltene Leiden “ (Synonyme englisch: orphan drugs, orphan pharmaceutical drug, orphan medicinal product) bezieht sich die Bezeichnung auf die oben aufgeführte Definition seltener Erkrankungen mit für verschiedene Regionen unterschiedlich geltenden Prävalenzen. Neben der Definition für seltene Erkrankungen auf der Basis der Prävalenz gehört dazu auch die fehlende kommerzielle Rentabilität.

Orphan-Arzneimittel sind wegen des meist kleinen oder sehr kleinen Marktes und des damit verbundenen geringen Umsatzes während des gesetzlichen Patentschutzes - bei gleichzeitig hohen Entwicklungskosten - für die pharmazeutische Industrie nicht interessant. Anstelle eines kleinen Marktes betrifft dies prinzipiell auch Arzneimittel mit einem Markt mit geringer Kaufkraft, typisch für häufige Krankheiten in ärmeren Ländern und hier v. a. Tropenkrankheiten, die aber nicht Gegenstand dieses Artikels sind.

Von politischer Seite wurde zur Förderung und Entwicklung derartiger Arzneimittel 1983 in den USA der „Orphan Drug Act“(ODA) erlassen [6].

Der hauptsächliche Anreiz für die Pharmazeutischen Unternehmen ist das exklusive Recht der Vermarktung (ODA: 7 Jahre). Zusätzlich werden über den ODA Forschungsmittel für Unternehmen und akademische Forschergruppen zur Verfügung gestellt, reduzierte Gebühren im Zulassungsprozess und Steuerminderungen gewährt, während Arzneimittel für seltene Erkrankungen bezüglich ihres therapeutischen Potenzials bewertet werden.

Zu den im Rahmen des Ophan Drug Acts von der US Food and Drug Administration (FDA) verabschiedeten „Orphan Drug Designation“ Programmen gehören das „Humanitarian Use Device (HUD) Designation“ Programm, das „Orphan Products Clinical Trials Grants“ Programm, das „Pediatric Device Consortia (PDC) Grant“ Programm und das „Orphan Products Natural History Grants“ Programm.

Seit ihrer Einführung haben diese Verordnungen zu einer deutlichen Steigerung im Bereich der Entwicklung von Arzneimitteln für seltene Erkrankungen geführt. In den USA betrifft dies die Markteinführung von mehr als 600 „Orphan Drugs“ [7].

Die Europäische Union hat im Jahr 2000 die „Verordnung über Arzneimittel für seltene Leiden “ in Kraft gesetzt. Ziel dieser Verordnung ist es, „ein Gemeinschaftsverfahren für die Ausweisung von Arzneimitteln als Arzneimittel für seltene Leiden festzulegen und Anreize für die Erforschung, Entwicklung und das Inverkehrbringen von als Arzneimittel für seltene Leiden ausgewiesenen Arzneimitteln zu schaffen“.

Innerhalb der Europäischen Arzneimittelagentur (EMA) verabschiedet ein „Ausschuss für Arzneimittel für seltene Krankheiten (Committee for Orphan Medicinal Products, COMP)“ regelmäßig Empfehlungen zur Zuerkennung des Status „Arzneimittel für eine seltene Krankheit“.

Kriterien für die Ausweisung als Arzneimittel für seltene Leiden sind:

„...(1) Ein Arzneimittel wird als Arzneimittel für seltene Leiden ausgewiesen, wenn der Investor nachweisen kann, dass

a) das Arzneimittel für die Diagnose, Verhütung oder Behandlung eines Leidens bestimmt ist, das lebensbedrohend ist oder eine chronische Invalidität nach sich zieht und von dem zum Zeitpunkt 
der Antragstellung in der Gemeinschaft nicht mehr als 5 von zehntausend Personen betroffen sind, oder

das Arzneimittel für die Diagnose, Verhütung oder Behandlung eines lebensbedrohenden Leidens, eines zu schwerer Invalidität führenden oder eines schweren und chronischen Leidens in der Gemeinschaft bestimmt ist und dass das Inverkehrbringen des Arzneimittels in der Gemeinschaft ohne Anreize vermutlich nicht genügend Gewinn bringen würde, um die notwendigen Investitionen zu rechtfertigen, und

b) in der Gemeinschaft noch keine zufriedenstellende Methode für die Diagnose, Verhütung oder Behandlung des betreffenden Leidens zugelassen wurde oder dass das betreffende Arzneimittel - sofern eine solche Methode besteht - für diejenigen, die von diesem Leiden betroffen sind, von erheblichem Nutzen sein wird“ (zitiert aus: [8]).

Die Marktexklusivität für die zugelassene Indikation des im Gemeinschaftsregister der EU als Orphan-Arzneimittel eingetragenen Medikamentes gilt in Europa für die Dauer von 10 Jahren. Zusätzlich werden auch in Europa dem Pharmahersteller ein Teil der Zulassungsgebühren erlassen. Eine weitere erhebliche Kostenersparnis entsteht durch ein vereinfachtes Zulassungsverfahren.

Die in der EU zugelassenen Arzneimittel mit Orphan-Status sind in der Datenbank Orphanet abrufbar und werden im „Verzeichnis der Arzneimittel für seltene Krankheiten in Europa“ veröffentlicht.

Da eine Zulassung als Arzneimittel für eine seltene Erkrankung (Orphan-Arzneimittel) nur dann erteilt wird, wenn die Erkrankung selten ist und es hierfür bisher keine adäquate Therapie gibt, entfällt für ein Orphan-Arzneimittel in der Regel die normalerweise bestehende Notwendigkeit des Nachweises eines Zusatznutzens entsprechend des im Jahr 2010 verabschiedeten Gesetz zur Neuordnung des Arzneimittelmarktes (AMNOG). Allerdings betrifft diese Erleichterung nur Arzneimittel, die aufgrund ihrer Zulassung für seltene Erkrankungen einen geringen Umsatz haben und gilt nicht für pharmazeutische Unternehmer, die mit dem Orphan-Arzneimittel einen Umsatz von mehr als 50 Mio. Euro erzielen. In diesen Fällen muss der Nachweis des Zusatznutzens erbracht werden. Die Forderung nach dem Nachweis eines Zusatznutzen könnte einer der Gründe sein, warum die FDA im Vergleich zur EMA mehr onkologische Medikamente inbesondere für Subgruppen onkologischer Erkankungen mit größerer Prävalenz zugelassen hat [9].

Allerdings gibt es auch Kontroversen über „unerwünschte Nebenwirkungen “ der Regularien und Förderprogramme im Zusammenhang mit der Entwicklung von Arzneimitteln für seltene Erkrankungen. Zum Teil wird auch von Manipulation („gaming the system“) zur Gewinnmaximierung gesprochen [10]. Bei einer zunehmenden Anzahl von Zulassungen für „seltene Erkrankungen“ handelt es sich um Biomarker-definierte Subgruppen nicht-seltener Erkrankungen, insbesondere in der Onkologie [11].

Bereits seit einiger Zeit wird über das Phänomen der „SalamiTaktik“ bei nicht-seltenen Erkrankungen zum Zwecke der Medikamentenzulassung unter Nutzung der Marktvorteile im Zusammenhang mit dem „Orphan Drug“ Status berichtet $[12,13]$.

Geringere Forschungs- und Entwicklungskosten (kleinere klinische Studien oder Beobachtungsstudien), beschleunigte Begutachtungsprozesse bei den Zulassungsbehörden, reduzierter oder minimaler Wettbewerb und anderes haben dazu geführt, dass die „Orphan Drugs“ inzwischen zu den teuersten und profitabelsten
Medikamenten im Weltmarkt zählen [14]. Daher werden die Anreizsysteme überarbeitet werden müssen.

\section{Klinische Studien und biometrische Aspekte}

Der Entwicklung von Therapien für seltene Erkrankungen stehen auch zahlreiche Probleme aus biometrischer Sicht bei der Durchführung klinischer Studien als Voraussetzung für die Zulassung von Therapien entgegen. Diese umfassen die niedrige Krankheitsprävalenz, die Heterogenität von Patientenpopulationen, die geographische Dispersion und den hohen Anteil von Kindern. Die Patientenzahlen in klinischen Studien für seltene Erkrankungen sind in der Regel klein mit entsprechenden Herausforderungen für ein effizientes Studiendesign und spezielle biometrische Methoden für die Outcome-Analyse bei limitierten Populationen.

Ein wichtiger Aspekt der ethischen Beurteilung klinischer Studien generell ist jedoch die biometrische Qualität mit Hinblick auf Studiendesign, Fallzahlberechnung und statistische Analyse, da methodologisch schlechte Forschung am Menschen per se als unethisch zu werten ist [15].

Die meisten statistischen Design- und Analysemethoden für klinische Studien wurden für Studienpopulationen von mindestens mehreren hundert Patienten entwickelt und evaluiert. Diese Methoden sind aber nicht unbedingt geeignet, um Therapien zu beurteilen, wenn die Stichprobengröße - wie z. B. bei seltenen Erkrankungen naturgemäß - klein ist. Ein spezifischer Grenzwert („Cut off“) für die Stichprobengröße, bei der Standardmethoden nicht mehr als adäquat betrachtet werden können, ist nicht genau bekannt. Daher werden in gegenwärtigen Untersuchungen statistische Methoden evaluiert, um eine hohe Aussagefähigkeit auch bei kleinen Studienpopulationen zu gewährleisten.

Zu diesen Methoden gehört z. B. die Aufnahme multipler bzw. zusammengesetzter Endpunkte („Composite Endpoints“) für Schlussfolgerungen zu Therapieeffekten. Die Kombination von Behandlungseffekten aus verschiedenen Endpunkten kann die statistische Power der biometrischen Tests verbessern [16, 17].

Eine weitere Möglichkeit, den im Kontext seltener Erkrankungen naturgemäßen Problemen randomisierter klinischer Studien, die immer noch den Goldstandard der klinischen Forschung darstellen, zu begegnen, sind „Real World Evidence“ (RWE)-Studien. Reale Evidenz in der Medizin bedeutet Evidenz aus Beobachtungsdaten, die außerhalb des Kontextes randomisierter kontrollierter Studien, während der routinemäßigen klinischen Praxis gewonnen werden. Die Analyse von „Real Word“-Daten können die Nutzenbewertung von Arzneimitteln auch im Kontext seltener Erkrankungen unterstützen. Die durch fortschreitende Digitalisierungsprozesse verbesserte Datenverfügbarkeit $[18,19]$ lässt erwarten, dass „Real Word Evidence“ zukünftig eine zunehmende Rolle auch bei der Entwicklung, der Nutzenbewertung und bei den regulatorischen Prozessen (Zulassung) von Therapien für seltene Erkrankungen spielen wird [20-23].

Auch wenn die üblichen Richtlinien für die Durchführung klinischer Studien (z. B. ICH Guidelines) auch für klinische Studien bei seltenen Erkrankungen gelten, haben die Europäische Arzneimittelbehörde (EMA) und der Ausschuss für Humanarzneimittel (Committee for Medicinal Products for Human Use, CHMP) spezielle Richtlinien für die Durchführung klinischer Studien in kleinen Stu- 
dienpopulationen entwickelt und veröffentlicht: EMA/CHMP guideline on clinical trials in small populations[24].

Auch das „Internationale Konsortium zur Erforschung seltener Erkrankungen“ (,International Rare Diseases Research Consortium“, IRDiRC) hat mit einer Task Force für klinische Studien bei kleinen Populationen („Small Population Clinical Trials Task Force", SPCT) entsprechende Empfehlungen herausgegeben $[25,26]$.

\section{Ethische Aspekte}

Legt man die 4 medizinethischen Grundprinzipien von Beauchamp und Childress [27] zugrunde, dann müssen auch im Rahmen der Entscheidungsfindung und Gewichtung im Kontext seltener Erkrankungen das Recht auf Selbstbestimmung der Patienten (Prinzip der Autonomie), das Prinzip der Schadensvermeidung (Non-Malefizienz, „primum non nocere“), das Prinzip der Fürsorge (Hilfeleistung, Benefizienz) und das Prinzip der Gerechtigkeit betrachtet werden.

Die Entscheidung, wie viel eine Gesellschaft für die Erforschung seltener Erkrankungen und ihrer Therapie ausgeben sollte, stellt ein moralisches Dilemma dar. Einerseits betreffen diese definitionsgemäß zahlenmäßig nur einen geringen Teil der Individuen einer Gesellschaft. Von einem utilitaristischen Standpunkt aus wäre die Allokation erheblicher Ressourcen für seltene Erkrankungen moralisch falsch, da sie den aggregierten Gesamtnutzen, d. h. die Summe des Wohlergehens aller Individuen einer Gesellschaft, nicht maximiert. Andererseits wird es auch als moralische Verpflichtung der Gesellschaft angesehen, diejenigen, die unglücklicherweise von einer seltenen Erkrankung betroffen sind, für die es bisher keine Heilung gibt, nicht im Stich zu lassen. Zudem hat Medizin an sich die professionelle Verpflichtung zu wissenschaftlichem Fortschritt, d. h. das Wissen um die Krankheiten und deren Therapie zu mehren. Die verschiedenen Prinzipien kommen daher - auch je nach dem, von welchem Standpunkt aus sie betrachtet werden - bezüglich der "Gerechtigkeit" in Widerspruch zueinander [28].

Als jüngstes Beispiel mag die Diskussion um das bisher teuerste Medikament der Welt - Zolgensma (Onasemnogene Abeparvovec) - des Schweizer Pharmakonzerns Norvatis gelten. Die Gentherapie Zolgensma bremst den Muskelschwund bei schwerer spinaler Muskelatrophie SMA1. Ursache der spinalen Muskelatrophie ist ein erblicher homozygoter Defekt im SMN1-Gen. Durch die Gentherapie mittels eines adenoviralen Vektors wird mit Zolgensma eine funktionsfähige Variante des Gens SMN1 in die Motoneuronen des Rückenmarks integriert, die das Gen SMN1 für ihr Überleben und das Aufrechterhalten ihrer Funktion benötigen. Die Erkenntnisse über den Nutzen von Zolgensma basieren v. a. auf einer kleinen Studie mit 12 Kindern, von denen die Mehrheit die Kopfkontrolle erlangten und 2/3 selbständig sitzen konnten; 24 Monate nach der Behandlung waren alle noch am Leben [29]. Im Jahr 2020 hat die Europäische Arzneimittelbehörde (EMA) die Zulassung für das Medikament erteilt. Die einmalige Gentherapie soll circa 2 Mio. Euro kosten, wobei die durchaus umstrittene Art der Preisbildung als „value-based pricing“ bezeichnet wird, da einmalige, an der genetischen Ursache ansetzende Therapien nach Argumentation der Hersteller eine andere Betrachtungsweise als chronische Therapien erfordern. In Deutschland wird die Gentherapie in den kommenden Monaten den erforderlichen Nutzenbewertungsprozess nach dem Gesetz zur Neuordnung des Arzneimittelmarktes (Arzneimittelmarktneuordnungsgesetz, AMNOG) durchlaufen. Von besonderer medizinethischer, politischer und medizin-ökonomischer Bedeutung ist die Tatsache, dass das Medikament ursprünglich in gemeinnützigen und spendenfinanzierten Laboren in den USA und Frankreich entstanden ist [30].

Der utilitaristische Standpunkt im Sinne der Maximierung des Gemeinwohls bildet die Grundlage einer ökonomischen Evaluation zur Priorisierung in der Gesundheitspolitik. Allerdings gibt es keinen vollständigen Konsens darüber, welche Werte „maximiert“ werden sollen. Ein übliches Maß sind hier die sogenannten „verlorenen gesunden Lebensjahre " (behinderungs- bzw. krankheitskorrigierte Lebensjahre; englisch: „disability-adjusted life years“ oder „disease-adjusted life years“, DALY). Das DALY-Konzept wurde erstmals im Weltentwicklungsbericht 1993 von der Weltbank präsentiert. Mit diesem Konzept soll die Bedeutung verschiedener Krankheiten auf die Gesellschaft einschließlich der Effizienz von Interventionen (Vorbeugung und Behandlung) als "Geldeinheit pro gewonnenes DALY“" messbar werden [31].

Neben dem Maximierungs-Prinzip gibt es dabei auch das Bedürftigkeits- und das Egalitätsprinzip. Außerdem muss berücksichtigt werden, dass die einzelnen seltenen Erkrankungen per Definition nicht häufig vorkommen, die Gesamtheit der seltenen Erkrankungen aber relativ hoch ist. Daher formulieren Gericke und Ko-Autoren 2 wesentliche Fragen [28]:

1.Welcher Umfang von Ressourcen sollte der Erforschung seltener Erkrankungen und ihrer Therapien zugeordnet werden?

2. Welcher Umfang von Ressourcen sollte jeder einzelnen seltenen Erkrankungen zugeordnet werden?

Weitere Probleme bei der Ressourcenallokation für Forschungszwecke entstehen im Zusammenhang mit einer ökonomischen Beurteilung aus der extremen Unsicherheit des Erfolgs bzw. Nutzens. Der minimale Markt, um das Interesse der Industrie an der Entwicklung eines „Orphan Drugs“ zu wecken, wird mit 100 Mio. US Dollar eingeschätzt [32], liegt aber wahrscheinlich viel höher.

Neben dem utilitaristischen Standpunkt besteht der z. B. auch in der Charta der Grundrechte der EU verankerte rechtliche Anspruch auf einen Zugang zur medizinischen Versorgung und das Recht auf medizinische Leistung unter den jeweiligen nationalen Gesetzen. Allerdings betrifft dies im engeren Sinne nicht das Recht bzw. den Anspruch auf Forschungsförderung für bisher nicht-existierende Therapien [28].

Im Bereich des Prinzips der Fürsorge (Hilfeleistung, Benefizienz) gelten für die Akteure sowohl die Erfordernisse der Zurverfügungstellung als auch die Abwägung und das Balancieren von Leistung, Risiko und Kosten.

Das Konzept der Nichtaufgabe bzw. des Nicht-im-Stich-Lassens (non-abandonment) trifft auch für den Bereich der seltenen Erkrankungen zu, da sie - zumindest ohne künstliche staatliche Anreizsysteme - aufgrund fehlender oder nur geringer Marktanreize von Forschung und Entwicklung vernachlässigt werden. Die entsprechenden, in diesem Artikel beschriebenen politischen und gesellschaftlichen Anstrengungen auf verschiedenen Ebenen, die alle darauf abzielen, das Wissen um seltene Krankheiten zu erweitern und die Diagnose, Versorgung und Behandlung von Patienten mit seltenen Krankheiten zu verbessern, sind im Kontext des „non-abandonment“ zu bewerten. 
Die verschiedenen moralischen Verpflichtungen verlangen nach einer (finanziellen) Unterstützung der Erforschung seltener Erkrankungen und ihrer Therapie einschließlich der Entwicklung von Orphan Drugs auf unterschiedlichen Ebenen [28].

Dass die bisherigen politischen und gesellschaftlichen Aktivitäten wirksam waren, lässt sich unzweifelhaft an der seit ihrer Einführung deutlich gewachsenen Anzahl an zugelassenen Medikamenten zur Behandlung seltener Erkrankungen ablesen [7].

Dennoch bleiben viele ethische Aspekte und Herausforderungen im Bereich seltener Erkrankungen ungelöst, die z. T. auch auf unterschiedlichen Normen und Regeln in verschiedenen Ländern beruhen. Diese betreffen insbesondere auch internationale (mit Ausnahme sehr positiver Ansatzpunkte in der Europäischen Union) und gobale Herangehensweisen. Eine wichtige Rolle für die Gewichtung und Lösung ethischer Dilemmata sowie für die faire Ressourcenverteilung nimmt auch die Zusammensetzung der Entscheidungsgremien ein [33]. Auch aufgrund der Fortschritte in der DNA-Sequenzierung, aktueller Entwicklungen in der Genom-Editierung (Genome Editing oder Genomchirurgie), z. B. mittels der CRISPR/Cas-Methode sowie der in vivo-Gentherapie mittels adenoviraler Vektoren, werden die medizinethischen Herausforderungen auch im Bereich seltener Erkrankungen zukünftig weiter wachsen [34-37].

\section{Juristische Aspekte und ambulante spezial- fachärztliche Versorgung ${ }^{1}$}

Zum Thema „seltene Erkrankungen“ ist wenig juristisch geregelt. Lediglich in $\S 116$ b Abs. 1 S. 2 Nr. 2 SGB V - im Rahmen der Regelung zur ambulanten spezialfachärztlichen Versorgung - werden seltene Erkrankungen explizit genannt. Da die Definition für Seltene Erkrankungen nur auf die Prävalenz abstellt, besteht keine Einschränkung auf schwere Verlaufsformen. Daher sind auch Verdachtsdiagnosen ausreichend für den Zugang zu einer spezialfachärztlichen Behandlung [38].

Relevant werden seltene Erkrankungen außerdem beim sogenannten Off-Label-Use, d. h. bei einer Anwendung von Arzneimitteln in Indikationsgebieten, auf die sich die Zulassung nicht bezieht. Eine solche Anwendung ist - jedenfalls im Rahmen der gesetzlichen Krankenversicherung - nur in Ausnahmefällen zulässig. Das Vorliegen einer extrem seltenen, nicht systematisch erforschbaren $\mathrm{Er}$ krankung stellt einen solchen Ausnahmefall dar (ständige Rechtsprechung des BSG, vgl. BSG, Urt. v. 19.3.2002 - B 1 KR 37/00 R, NJW 2003, 460). Siehe dazu außerdem den sogenannten „Nikolaus-Beschluss“ des Bundesverfassungsgerichts. Hier heißt es im Leitsatz: „Es ist mit den Grundrechten aus Art. 2 Abs. 1 GG in Verbindung mit dem Sozialstaatsprinzip und aus Art. 2 Abs. 2 Satz 1 GG nicht vereinbar, einen gesetzlich Krankenversicherten, für dessen lebensbedrohliche oder regelmäßig tödliche Erkrankung eine allgemein anerkannte, dem medizinischem Standard entsprechende Behandlung nicht zur Verfügung steht, von der Leistung einer

\footnotetext{
${ }^{1}$ Der Abschnitt „Juristische Aspekte“ wurde im Wesentlichen zusammengestellt von Dr. iur. Carina Dorneck, M.mel., Juristischer Bereich, Juristische und Wirtschaftswissenschaftliche Fakultät der Martin-Luther-Universität Halle-Wittenberg
}

von ihm gewählten, ärztlich angewandten Behandlungsmethode auszuschließen, wenn eine nicht ganz entfernt liegende Aussicht auf Heilung oder auf eine spürbare positive Einwirkung auf den Krankheitsverlauf besteht“. (BVerfG, Beschl. v. 6.12.2005-1 BvR 347/98, NJW 2006, 891). Diesen Vorgaben entsprechend ordnet auch $\S 31$ Abs. 1 S. 4 SGB V an, dass der Vertragsarzt Arzneimittel, die eigentlich von der Versorgung ausgeschlossen sind, ausnahmsweise in medizinisch begründeten Einzelfällen verordnen kann $[39,40]$.

Weiterhin stellt sich die Frage nach der Haftung bei Nichterkennen einer seltenen Erkrankung. Hierzu hat die Rechtsprechung entschieden, dass ein (Krankenhaus-)Arzt für Diagnosefehler bei Erkrankungen, die im Alltag eines durchschnittlichen Krankenhauses praktisch nicht anzutreffen sind, nicht haftet (OLG Koblenz, Beschl. v. 27.1.2014-5 U 1383/13, MedR 2015, 38 ff.).

\section{Nationales Aktionsbündnis für Menschen mit seltenen Erkrankungen (NAMSE)}

Das Nationale Aktionsbündnis für Menschen mit seltenen Erkrankungen (NAMSE) wurde im Jahr 2010 als Zusammenschluss zwischen dem Bundesministerium für Gesundheit (BMG), dem Bundesministerium für Bildung und Forschung (BMBF) und ACHSE e.V. (Allianz Chronischer seltener Erkrankungen) mit 25 Bündnispartnern (ausschließlich Spitzen- und Dachverbände der wesentlichen Akteure im Gesundheitswesen) durch die Annahme einer gemeinsamen Erklärung als Koordinierungs- und Kommunikationsgremium gründet, mit dem Ziel, auf der Grundlage bereits bestehender Strukturen und europäischer Erfahrungen eine bessere Patientenversorgung für Menschen mit seltenen Erkrankungen auf den Weg zu bringen. Dazu bündelt es bestehende Initiativen, vernetzt Forscher und Ärzte und führt Informationen für Ärzte und Patienten zusammen (nach: www.namse.de).

Seit 2011 beteiligt sich auch das Bundesministerium für Bildung und Forschung (BMBF) mit seiner Förderung nationaler Verbünde für seltene Erkrankungen an dem „International Rare Diseases Research Consortium (IRDiRC)“, welches von der EU-Kommission und dem US National Institute of Health (NIH) gegründet wurde. Mehr als 50 internationale Partner - darunter Forschungsförderer wie das BMBF, Patientenverbände und Industriepartner - beteiligen sich an dem IRDiRC-Konsortium.

Im gleichen Jahr wurde mit dem GKV-Versorgungsstrukturgesetz (GKV-VStG) unter der Bezeichnung „Ambulante spezialfachärztliche Versorgung“ (ASV) im §116b SGB V ein neuer Versorgungsbereich geschaffen, in dem Vertragsärzte und Krankenhausambulanzen nach einheitlichen Rechtsvorschriften Patienten versorgen. Um für Betroffene und ihre Angehörigen sowie für medizinisches, therapeutisches und pflegerisches Personal Wissen zu seltenen Erkrankungen qualitätsgesichert bereitzustellen, wurde im Jahr 2014 das zentrale Informationsportal seltene Erkrankungen ZIPSE (www. portal-se.de) aufgebaut. Dieses stellt selbst keine Primärinformationen bereit, sondern verweist auf bereits existierende, qualitätsgesicherte Angebote über seltene Erkrankungen wie Orphanet Deutschland, Allianz Chronischer Seltener Erkrankungen (ACHSE), SE-Atlas oder Zentren für seltene Erkrankungen. 
Der Nationale Aktionsplan mit 52 Maßnahmenvorschlägen, um das zukünftige Handeln des deutschen Gesundheits- und Sozialsystems im Kontext von seltenen Erkrankungen zu koordinieren und zu organisieren, wurde von den Bündnispartnern des NAMSE im Jahr 2013 veröffentlicht. Er beschreibt auch ein 3-stufiges Zentrenmodell. Die Kriterien für Typ-A-Zentren (Referenzzentren) und Typ-B-Zentren (Fachzentren) wurden durch eine Arbeitsgruppe des NAMSE konkretisiert und operationalisiert und nach Zustimmung durch die Bündnispartner 2014 veröffentlicht.

Ein Bestandteil des Nationale Aktionsbündnis für Menschen mit seltenen Erkrankungen (NAMSE) ist das Projekt OSSE (Open-Source-Registersystem für seltene Erkrankungen / Open Source Registry System for Rare Diseases), gefördert vom Bundesministerium für Gesundheit.

Ziel des Projekts ist es, mittels OSSE Patientenvereinigungen, Klinikern, Forschern und anderen Beteiligten mithilfe einer Open-Source-Software den Aufbau von Patientenregistern zu ermöglichen. Dies soll zu einer Stärkung der nationalen Registerlandschaft führen. Dabei wurde im Rahmen von OSSE ein Minimaldatensatz entwickelt, welcher inzwischen durch eine Version von Seiten der EU abgelöst worden ist. Dieser sollte von allen teilnehmenden Registern abgedeckt werden, um die Auffindbarkeit von und den Austausch mit Registern zu ermöglichen und zu fördern (www.osse-register.de).

Seit 2009 haben sich deutschlandweit an Universitätskliniken Zentren konstituiert (Zentren für seltene Erkrankungen, ZSE), die sich der Versorgung von Patienten mit seltenen Erkrankungen verschrieben haben. Eine Maßnahme aufgrund des Nationalen Aktionsplans für Menschen mit Seltenen Erkrankungen NAMSE ist die Zurverfügungstellung des Versorgungsatlas für Menschen mit seltenen Erkrankungen (se-atlas). Auf diesem Internetportal können sich Betroffene, Angehörige und Ärzte, aber auch nicht-medizinisches Personal und die breite Öffentlichkeit einen umfassenden Überblick über Versorgungsmöglichkeiten und Selbsthilfeorganisationen für Menschen mit seltenen Erkrankungen in Deutschland verschaffen. Hier werden Links zur Verfügung gestellt für

- Zentren für seltene Erkrankungen (ZSE) in Deutschland,

- eine Übersicht der übergeordneten Einrichtungen,

- eine Übersicht zertifizierter Einrichtungen und

- eine Übersicht über Europäische Referenznetzwerke (ERN), (www.se-atlas.de).

Für die Hals-Nasen-Ohrenheilkunde, Kopf- und Hals-Chirurgie ist unter anderem von Bedeutung das Europäische Referenznetzwerk für kraniofaziale Anomalien und Ohren-, Nasen-, Halsstörungen (https://ern-cranio.eu/).

Das Thema der seltenen Erkrankungen wurde auch im Nationalen kompetenzbasierten Lernzielkatalog Medizin (NKLM) und Zahnmedizin (NKLZ), in der neuen Approbationsordnung für Ärzte (ÄApprO), im GKV-Versorgungsstärkungsgesetz (GKV-VSG) bezüglich der Weiterentwicklung der Ermächtigungsregelung für Hochschulambulanzen ( $§ 117$ SGB V) und im Krankenhausstruktur-Gesetz (KHSG) mit Berücksichtigung von besonderen Zentrumsaufgaben für Krankhäuser aufgrund von besonderen Vorhaltungen berücksichtigt. Der Gemeinsame Bundesausschuss (G-BA) hat beschlossen, dass die besonderen Aufgaben über Zentrumszuschläge zu finanzieren sind, da es sich um Leistungen für Patientinnen und Patienten anderer Krankenhäuser handelt (z. B. interdisziplinäre
Fallkonferenzen) oder um übergreifende Aufgaben (z. B. Registerführung und -auswertung). Bereits im Jahr 2016 wurde die „Hochdurchsatzsequenzierung“ unter anderem zur Diagnostik von Seltenen Erkrankungen in den Einheitlichen Bewertungsmaßstab (EBM) aufgenommen. Mit dem Musterdatensatz 2020 hat das DIMDI standardisierte Kodes für die Kodierung von seltenen Erkrankungen veröffentlicht. Diese enthalten neben dem Alpha-ID-Kode und dem ICD-10-GM-Kode auch die sogenannte Orpha-Kennnummer aus Orphanet. Im letzten Jahr hat das BfArM die Alpha-ID-SE Version 2021 veröffentlicht, die für einen großen Teil der Einträge zu seltenen Erkrankungen zusätzlich die jeweilige Orpha-Kennnummer, die aus der Datenbank „Orphanet“ für seltene Erkrankungen stammt, enthält. Die Version 2021 enthält 8043 Diagnosenbezeichnungen mit Orpha-Kennnummer.

Ein weiteres als Teil des Nationalen Aktionsplans für Menschen mit seltenen Erkrankungen (NAMSE) vom Bundesministerium für Gesundheit ins Leben gerufenes Projekt ist das Zentrale Informationsportal über seltene Erkrankungen (ZIPSE). Das Projekt wurde Ende 2019 von der Stiftung Gesundheitswissen übernommen. „...Hier finden Betroffene und Angehörige eine Übersicht medizinischer, therapeutischer und pflegerischer Leistungserbringer im Bereich seltener Erkrankungen. Dabei werden Informationen, insbesondere zu Diagnostik, Therapie, Selbsthilfe, Versorgungseinrichtungen, Forschung und Registern anhand spezifischer Kriterien qualitätsgesichert gebündelt. Zudem finden sich auf diesem Portal Ansprechpartner und Informationsmöglichkeiten zu sozial- und leistungsrechtlichen Fragen. Das ZIPSE-Informationsportal versteht sich als Wegweiser und verweist auf qualitätsgesicherte Informationsquellen im Netz....“ (zitiert aus: https://www.portal-se.de/ziele).

\section{Allianz Chronischer seltener Erkrankungen - ACHSE e.V}

Die ACHSE e.V. ist ein Netzwerk von Selbsthilfeorganisationen. Sie „tritt als Sprachrohr, Multiplikator und Vermittler auf. Sie sensibilisiert für die Belange von Menschen mit seltenen Erkrankungen und ihre spezifischen Probleme. Sie fördert das Wissen über diese Erkrankungen in der Bevölkerung, bei Interessenvertretern, aber auch bei Ärzten und Therapeuten“.

Zu den Aufgabenbereichen der ACHSE zählen: Betroffene und Angehörige zu unterstützen, Ärzte und Therapeuten zu vernetzen, Forschungsförderung anzuregen, die Öffentlichkeit zu sensibilisieren, politische Interessen zu vertreten und das Informations- und Wissensmanagement zu verbessern (zitiert nach: www.achse-online.de).

ACHSE e.V. beteiligt sich u. a. auch am jährlichen „Rare Disease Day“, dem internationalen Tag der seltenen Erkrankungen am letzten Tag im Februar (www.rarediseaseday.org). Diese Kampagne richtet sich primär an die allgemeine Öffentlichkeit, Politiker und Gesundheitspolitker, Repräsentanten der Industrie, Forschende, Therapeuten und alle Interessierten an seltenen Erkrankungen.

\section{Forschung und Forschungsförderung}

Durch die Erforschung seltener Erkrankungen können grundlegende biomedizinische Zusammenhänge erforscht und aufgedeckt 
werden, deren Verständnis auch für die Aufklärung von Ursachen häufiger Erkrankungen essentiell ist.

Bereits im Jahr 1657 schrieb William Harvey that „....Nature is nowhere accustomed more openly to display her secret mysteries than in cases where she shows traces of her workings apart from the beaten path; nor is there any better way to advance the proper practice of medicine than to give our minds to the discovery of the usual law of $\mathrm{Na}$ ture by careful investigation of cases of rarer forms of disease...." [41]. Forscher, Pharmaunternehmen und Investoren haben erkannt, dass die wissenschaftlichen Einblicke und Erkenntnisse bei der Erforschung seltener Erkrankungen sich auch mit Hinblick auf die häufigeren Krankheiten und das Wissen um deren Krankheitsmechanismen und Therapien auszahlen. Der hauptsächliche Grund dafür ist, dass viele seltene Erkrankungen Resultate einzelner Genveränderungen sind, die es Wissenschaftlern ermöglichen, die Folgen der Gendefekte wie in einem gut kontrollierten Experiment zu beobachten. Ein gutes Beispiel ist die Erforschung der familiären Hypercholesterinämie, die zur Entwicklung der Statine führte (nach: [28]).

Circa die Hälfte der in Deutschland über verschiedenste Förderinstitutionen geförderten Projekte aus dem Bereich seltener $\mathrm{Er}$ krankungen beschäftigt sich mit seltenen Krebserkrankungen. An zweiter Stelle stehen seltene genetische Erkrankungen. Umfassende Angaben dazu, wie viele Mittel überhaupt in Deutschland in die Erforschung seltener Erkrankungen fließen und auch eine systematische Erfassung aller Forschungsaktivitäten existiert derzeit nicht.

Für die klinische, patientenorientierte Erforschung seltener Erkrankungen besonders wichtig ist, in vernetzten überregionalen oder internationalen Strukturen zu arbeiten. Dabei nehmen Krankheitsregister eine zentrale Rolle ein. Diese können unter Berücksichtigung klarer Qualitätskriterien bezüglich Vollständigkeit und Vollzähligkeit als Basis für die Generierung von Evidenz durch hochwertige, klinische, v. a. nicht randomisierte Studien dienen.

Das Bundesministerium für Bildung und Forschung (BMBF) fördert seit 2003 nationale Forschungsverbünde für seltene Erkrankungen. Im Vordergrund der Arbeit der Forschungsverbünde steht die Aufklärung von Krankheitsursachen sowie Diagnose- und Therapieforschung. Diese decken ein breites Spektrum von seltenen Erkrankungen ab: Immunologie, Entwicklungsstörungen, Nierenerkrankungen, Erkrankungen des Nervensystems und Stoffwechselerkrankungen. Die Zielsetzung der geförderten Vorhaben ist insgesamt auf Translation ausgerichtet. Im Februar 2018 veröffentlichte das BMBF erneut eine Richtlinie zur Förderung translationsorientierter Verbundvorhaben im Bereich der seltenen Erkrankungen.

„Das BMBF und die Deutsche Forschungsgemeinschaft DFG beteiligen sich am European Joint Programmes on Rare Diseases (EJP RD). In diesem Programm arbeiten mit finanzieller Unterstützung der EU 130 Institutionen aus 35 Ländern von 2019 bis 2024 zur Schaffung eines umfassenden, nachhaltigen Forschungs-Ökosystems zusammen, das eine verbesserte Koordination und Rückkopplung zwischen Forschung, Krankenversorgung und medizinischer Innovation ermöglichen soll. Neben dem koordinierten Zugang zu Daten, Trainingsaktivitäten und der Beschleunigung von Translation ist die internationale Forschungsförderung ein wichtiger Bestandteil dieses Programms. Diese beinhaltet die Förderung von Vernetzungstreffen zum Wissensaustausch, der Kooperation von Industrie und Akademia zur Lösung spezifischer ForschungsHerausforderungen sowie von transnationalen Forschungsverbün- den. Bei letzteren bauen die beteiligten 31 Forschungsförderer aus 23 Ländern ihre in „E-Rare“ begonnenen gemeinsamen Aktivitäten aus. BMBF und DFG beteiligten sich an den ersten beiden Bekanntmachungen 2019 und 2020. Darüber hinaus sind 2 weitere Bekanntmachungen geplant (zitiert nach: www.namse.de)“.

Der Innovationsfonds des Gemeinsamen Bundesausschusses (G-BA) wird im laufenden Jahr Leitlinienprojekte zu seltenen Erkrankungen fördern. Die Förderung erfolgt dabei über 2 Kanäle: Zum einen fördert der Innovationsfonds die Erstellung oder Aktualisierung kompletter Leitlinien, zum anderen werden bei einzelnen klinisch relevanten Fragestellungen in Leitlinien auch Evidenzrecherchen des Institutes für Qualität und Wirtschaftlichkeit in der Medizin (IQWiG) unterstützt.

Das „Internationale Konsortium zur Erforschung seltener Erkrankungen“ („International Rare Diseases Research Consortium“, IR$\mathrm{DiRC}$ ) vereint staatliche und gemeinnützige Organisationen, pharmazeutische und biotechnologische Unternehmen sowie Dachorganisationen für Patienteninteressen und Wissenschaftler mit dem Ziel, die Erforschung seltener Erkrankungen weltweit voranzutreiben. Im Jahr 2017 hat das IRDiRC eine Vision für die Erforschung seltener Erkrankungen formuliert: Bis zum Jahr 2027 sollen alle Patientinnen und Patienten, die an einer bekannten seltenen Erkrankung leiden, innerhalb eines Jahres nach der ersten ärztlichen Behandlung eine genaue Diagnose, Versorgung und verfügbare Therapie erhalten. Darüber hinaus sollen 1000 neue Therapieansätze zugelassen werden - insbesondere für jene seltenen Erkrankungen, für die bis heute Behandlungsoptionen fehlen [42].

Desweiteren stellen auch verschiedene Stiftungen Fördermittel zur Erforschung seltener Erkrankugen bereit. Als Besipiel sei hier die Eva Luise und Horst Köhler Stiftung für Menschen mit Seltenen Erkrankungen (www.elhks.de) genannt, welche sich durch gezielte Forschungsförderung für eine bessere medizinische Versorgung von Menschen mit seltenen Erkrankungen einsetzt. Die Stiftung adressiert v. a. wesentliche Herausforderungen in den Bereichen neuer Zugänge zu qualitätsgesicherter, insbesondere molekulargenetischer Diagnostik, der Beschleunigung der Entwicklung spezifischer Therapien und den Ausbau von Experten-Netzwerken. Der jährlich vergebene Eva Luise Köhler Forschungspreis gehört mittlerweile zu den etabliertesten Auszeichnungen in diesem Forschungsbereich.

\section{Fazit}

Seltene Erkrankungen stellen besondere Herausforderungen an die Bereiche der Diagnostik, der präklinischen und klinischen Forschung, des interdisziplinären und multiprofessionellen Managements sowie der adäquaten Finanzierung einschließlich der Abrechnung und Kodierung sowie an regulatorische Aspekte. Sie führen zu besonderen Anforderungen an das Arzt-Patienten-Verhältnis, an die Nachsorge und die Kommunikation mit den mitbehandelnden Ärzten. Seltene Erkrankungen stellen außerdem eine medizinethische Herausforderung dar, insbesondere, wenn es um die Frage der Allokation in der Versorgung und der Erforschung geht. Von großer Bedeutung sind das Informationsmanagement und die Öffentlichkeitsarbeit, aber auch Aus-, Fort- und Weiterbildung sowie bewusste Forschungsförderung einschließlich der nationalen und internationalen Vernetzung, die Schaffung und Pflege von 
Registern und die klinische Zentrenbildung. Eine wichtige Rolle spielt die Selbsthilfe für Betroffene und Angehörige. Patienten und Angehörige sind häufig Wissensträger auf dem Gebiet ihrer jeweiligen Erkrankung und können helfen, dieses Wissens mit der Expertise der Leistungsträger zu verknüpfen.

Durch die politisch, akademisch und die Selbsthilfe gesteuerten Aktivitäten wurden in den letzten Jahren unter Beteiligung der verschiedenen Interessengruppen (Stakeholder) mit Blick auf die vielfältigen Herausforderungen gute Erfolge erzielt. Viele Herausforderungen bleiben jedoch bestehen und verlangen unsere besondere Aufmerksamkeit und unser besonderes Engagement, damit ohne unnötigen Zeitverzug seltene Erkrankungen diagnostiziert werden und Patienten Zugang zu einer adäquaten Behandlung an geeigneten Zentren erhalten.

\section{Danksagung}

Ich bedanke mich bei folgenden Kolleginnen und Kollegen für wertvolle Hinweise und Kommentare: Professores Jan Schildmann, Rafael Mikolajczyk, Walther Wohlgemuth, Henning Rosenau und Dr. iur. Carina Dorneck, M.mel..

\section{Interessenkonflikt}

S.K. Plontke gibt folgenden Interessenkonflikt an: Beratungstätigkeiten: AudioCure Pharma GmbH, Berlin; Astellas Global Development, Inc., Northbrook, IL Reisekostenerstattung bei Vortragstätigkeit: MED-EL Österreich und MED-EL Deutschland; Vortragshonorare: MED-EL Deutschland Forschungsprojekte: MED-EL Österreich und MED-EL, Deutschland; Oticon Medical, Dänemark; Cochlear Ltd., Australien; BMBF (Förderung klinische Studie zu Hörsturz); Vortragshonorare und Reisekostenerstattung: BV-HNO e.V.; Merck Serono, Darmstadt; Infectopharm, Heppenheim, Deutschland

Literatur

[1] Krause F. Shared Decision Making bei seltenen Erkrankungen. Ethik Med 2019; 31: 131-141

[2] Bundesgesundheitsministerium. Gesundheitsgefahren. Seltene Erkrankungen. In: https://www.bundesgesundheitsministerium.de/ themen/praevention/gesundheitsgefahren/seltene-erkrankungen. html, abgerufen 25.10 .2020

[3] Enzyklopädie WDf. Seltene Krankheit. In: https://de.wikipedia.org/ wiki/Seltene_Krankheit\#cite_ref-1, abgerufen 25. Oktober 2020

[4] [Anonym]. Bundesinstitut für Arzneimittel und Medizinprodukte. Orphanet. Das Portal für seltene Krankheiten und Orphan Drugs. In: https://www.orpha.net/consor/cgi-bin/index.php abgerufen 25. Oktober 2020

[5] [Anonym]. Bundesinstitut für Arzneimittel und Medizinprodukte. Orphanet Deutschland. In: https://www.bfarm.de/DE/Forschung/ Orphanet/_node.html, abgerufen 25. Oktober 2020;

[6] [Anonym]. Orphan Drug Act of 1983; Pub L. No. 97, 414, 96 Stat. 2049. In

[7] Le TT. Incentivizing Orphan Product Development: United States Food and Drug Administration Orphan Incentive Programs. Adv Exp Med Biol 2017; 1031: 183-196

[8] „Vom“, DOI,
[9] Vokinger KN, Kesselheim AS. Application of orphan drug designation to cancer treatments (2008-2017): a comprehensive and comparative analysis of the USA and EU. BMJ Open 2019; 9: e028634

[10] Herder M. What Is the Purpose of the Orphan Drug Act? PLoS Med 2017; 14: e1002191

[11] Kesselheim AS, Treasure CL, Joffe S. Biomarker-Defined Subsets of Common Diseases: Policy and Economic Implications of Orphan Drug Act Coverage. PLoS Med 2017; 14: e1002190

[12] Loughnot D. Potential interactions of the Orphan Drug Act and pharmacogenomics: a flood of orphan drugs and abuses? Am J Law Med 2005; 31: 365-380

[13] Rai AK. Pharmacogenetic interventions, orphan drugs, and distributive justice: the role of cost-benefit analysis. Soc Philos Policy 2002; 19: 246-270

[14] Meekings KN, Williams CS, Arrowsmith JE. Orphan drug development: an economically viable strategy for biopharma R\&D. Drug Discov Today 2012; 17: 660-664

[15] Hasford J, Koch A. Ethical aspects of clinical trials in rare diseases. Bundesgesundheitsblatt Gesundheitsforschung Gesundheitsschutz 2017; 60: 556-562

[16] McMenamin M, Berglind A, Wason JMS. Improving the analysis of composite endpoints in rare disease trials. Orphanet J Rare Dis 2018; 13: 81

[17] Ristl R, Urach S, Rosenkranz G et al. Methods for the analysis of multiple endpoints in small populations: A review. J Biopharm Stat 2019; 29: 1-29

[18] Nateqi ], Lin S, Krobath $\mathrm{H}$ et al. From symptom to diagnosis-symptom checkers re-evaluated : Are symptom checkers finally sufficient and accurate to use? An update from the ENT perspective. HNO 2019; 67: 334-342

[19] Sonntag D. Artificial intelligence in medicine-the wrong track or promise of cure?. HNO 2019; 67: 343-349

[20] Beaulieu-Jones BK, Finlayson SG, Yuan W et al. Examining the Use of Real-World Evidence in the Regulatory Process. Clin Pharmacol Ther 2020; 107: 843-852

[21] Franklin JM, Pawar A, Martin D et al. Nonrandomized Real-World Evidence to Support Regulatory Decision Making: Process for a Randomized Trial Replication Project. Clin Pharmacol Ther 2020; 107: 817-826

[22] O'Donnell JC, Le TK, Dobrin R et al. Evolving use of real-world evidence in the regulatory process: a focus on immuno-oncology treatment and outcomes. Future Oncol 2021; 17: 333-347

[23] Wu J, Wang C, Toh S et al. Use of real-world evidence in regulatory decisions for rare diseases in the United States-Current status and future directions. Pharmacoepidemiol Drug Saf 2020; 29: 1213-1218

[24] [Anonym]. EMA/CHMP guideline on clinical trials in small populations. In 2006

[25] Day S, Jonker AH, Lau LPL et al. Recommendations for the design of small population clinical trials. Orphanet J Rare Dis 2018; 13: 195

[26] Jonker AHM, Lau A, Ando LPL, Baroldi Y, Bretz P, Burman F, Collignon CF, Hamdani O, Hemmings M, Hilgers RJ, Irony RD, Karlsson I, Kirschner M, Krischer J, Larsson JP, Leeson-Beevers K, Molenberghs K, O'Connor G, Posch D, Roes M, Schaefer KC, Scott F, Senn J, Stallard SJ, Thompson N, Torres A, Zohar F, Ayme S, Day S., S. Small Population Clinical Trials: Challenges in the Field of Rare Diseases. In; 2016

[27] Beauchamp TL, Childress JF. Principles of Biomedical Ethics. Oxford: Oxford University Press; 2001

[28] Gericke CA, Riesberg A, Busse R. Ethical issues in funding orphan drug research and development. J Med Ethics 2005; 31: 164-168 
[29] Lowes LP, Alfano LN, Arnold WD et al. Impact of Age and Motor Function in a Phase 1/2A Study of Infants With SMA Type 1 Receiving Single-Dose Gene Replacement Therapy. Pediatr Neurol 2019; 98: 39-45

[30] Grill M, Kempmann A. Zolgensma: Wie das teuerste Medikament entstand. In; 2020

[31] WHO. World Health Organisation. World Development Report. 1993; Investing in Health. In; 1993

[32] Pabst JY. Medicaments orphelins: quelques aspects juridiques, ethiques et economiques. Rev Epidemiol Sante Publique 2001; 49: 387-396

[33] Kacetl ], Maresova P, Maskuriy R et al. Ethical Questions Linked to Rare Diseases and Orphan Drugs - A Systematic Review. Risk Manag Healthc Policy 2020; 13: 2125-2148

[34] He X, Urip BA, Zhang Z et al. Evolving AAV-delivered therapeutics towards ultimate cures. J Mol Med (Berl) 2021; Feb 16: 1-25. 10.1007/ s00109-020-02034-2

[35] Mendell JR, Al-Zaidy SA, Rodino-Klapac LR et al. Current Clinical Applications of In Vivo Gene Therapy with AAVs. Mol Ther 2021; 29: 464-488
[36] Oppel F, Schurmann M, Shao S et al. Perspectives of genome editing in otorhinolaryngology. HNO 2019; 67: 184-189

[37] Singer DRJR K. What is ahead for health policy and technology in the 2020s? Health Policy Technol 2020; 9: 3-4

[38] Makoski K. Erste Erfahrungen mit der ambulanten spezialfachärztlichen Versorgung nach § 116b SGB V. Gesundheit und Pflege. 2017; 2. 47-52

[39] Freund A. Die zulassungsüberschreitende Anwendung von Humanarzneimitteln (Off-Label-Use) in Deutschland Pharmazeutische Unternehmer, Vertragsärzte, Patienten und Versicherte im Spannungsfeld von Arzneimittel-, und Sozialrecht sowie zivil- und strafrechtlicher Haftung. PharmR 2004; 8. 275-299

[40] Heinemann AK. Off-label Use von Diagnostika am Beispiel der MR-Kontrastmittel. Medizin Produkte Recht 2007; 10: 113-140

[41] Willis R. The Works of William Harvey. London: Sydenham Society; 1847

[42] Lochmuller H, Torrent IF], Le Cam Y et al. The International Rare Diseases Research Consortium: Policies and Guidelines to maximize impact. Eur J Hum Genet 2017; 25: 1293-1302 